\section{Commentary: Ready Player One: A new "reality?"}

\author{
Seth B. Krantz, MD
}

The past decade has brought a significant increase in the performance of pulmonary segmentectomies, including "complex" segmentectomies that involve the division of multiple intersegmental planes. ${ }^{1-4}$ With increasing use of lung cancer screening, this trend is not likely to abate. Successful completion of these operations can be challenging owing not only to their technical complexity but also to the difficulty in accurately identifying the correct anatomic segment on standard preoperative imaging. In their very interesting article in this issue of JTCVS Techniques, Sadeghi and colleagues ${ }^{5}$ describe a novel technique to aid the preoperative planning of these procedures. They have developed a virtual reality (VR)based tool that uses artificial intelligence to create fully segmented and colorized reconstructions of preoperative computed tomography scans. The use of artificial intelligence allows significant portions of the overall process to be automated, with a final product that can then be manipulated and studied by the surgeon in an immersive VR environment.

In this pilot study of 10 patients, the authors focused primarily on technical feasibility and preoperative workflow, along with some preliminary clinical data on the accuracy and impact of the software. They were able to achieve complete segmental maps inclusive of the arteries, veins, and bronchi and were able to manipulate these maps within the VR platform in all 10 patients. After VR evaluation of the 3-dimensional segmental models, the surgical plan was changed in 4 of 10 patients. Intraoperative anatomy corresponded to the VR images in $100 \%$ of the patients, though

\footnotetext{
From the Division of Thoracic Surgery, Department of Surgery, NorthShore University HealthSystem, Evanston, Ill and Department of Surgery, University of Chicago Pritzker School of Medicine, Chicago, Ill.

Disclosures: The author reports no conflicts of interest.

The Journal policy requires editors and reviewers to disclose conflicts of interest and to decline handling or reviewing manuscripts for which they may have a conflict of interest. The editors and reviewers of this article have no conflicts of interest.

Received for publication March 30, 2021; revisions received March 30, 2021; accepted for publication March 31, 2021; available ahead of print April 3, 2021.

Address for reprints: Seth B. Krantz, MD, Division of Thoracic Surgery, NorthShore University HealthSystem, 2650 Ridge Ave, Walgreen Suite 3507, Evanston, IL 60201 (E-mail: skrantz@northshore.org).

JTCVS Techniques 2021;7:322-3

2666-2507

Copyright (C) 2021 The Author(s). Published by Elsevier Inc. on behalf of The American Association for Thoracic Surgery. This is an open access article under the CC BY-NC-ND license (http://creativecommons.org/licenses/by-nc-nd/4.0/).

https://doi.org/10.1016/j.xjtc.2021.03.034
}

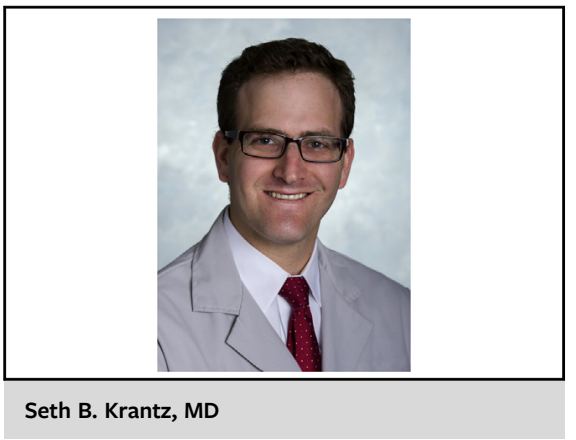

CENTRAL MESSAGE

Automated segmentation of standard computed tomography scans, coupled with an immersive VR environment is technically feasible and may improve our ability to perform complex segmental resections.

this was based on postprocedural surveys of the operating surgeon and not on any independent assessment. Both surgeons who used the system gave it high ratings for ease of use, overall usefulness, and likelihood of future use. Interestingly, both surgeons saw this as a supplement to conventional methods, not a replacement. Clinical outcomes were limited, but an R0 resection was achieved in all patients.

This very exciting study of a promising new technology is not without limitations. It is clearly a pilot study and lacks controls for interrater reliability, does not have a comparison with more standard 3-dimensional reconstruction techniques without segmentation and VR manipulation (or for that matter, with standard imaging techniques), and does not provide any true measure of how this tool impacts clinical outcomes. Future studies will need to compare patients who had more standard evaluation and VR-based segmental evaluation along with providing outcomes such as margin positivity, margin size, total lung segments removed, operative time, air leak, and others. Furthermore, a true blinded assessment of the VR accuracy is also not included in this early work.

As utilization of lung cancer screening expands, we will increasingly see small, subcentimeter tumors and a concomitant increase in segmental resection. Such resections will be further facilitated by future technological innovations, including VR simulation, enhanced robotics, augmented reality, and molecular enhanced imaging. Although there remains much work to do, the authors 
should be commended for helping push our field forward in pursuit of better outcomes for our patients.

\section{References}

1. Servais EL, Towe CW, Brown LM, Broderick SR, Block MI, Burfeind WR, et al. The Society of Thoracic Surgeons General Thoracic Surgery database: 2020 update on outcomes and research. Ann Thorac Surg. 2020;110:768-75.

2. Handa Y, Tsutani Y, Mimae T, Miyata Y, Imai K, Ito H, et al. Oncologic outcomes of complex segmentectomy: a multicenter propensity score-matched analysis. Ann Thorac Surg. 2021;111:1044-51.
3. Handa Y, Tsutani Y, Mimae T, Miyata Y, Okada M. Complex segmentectomy in the treatment of stage IA non-small-cell lung cancer. Eur J Cardiothorac Surg. 2020;57:114-21.

4. Suzuki K, Saji H, Aokage K, Watanabe SI, Okada M, Mizusawa J, et al. Comparison of pulmonary segmentectomy and lobectomy: safety results of a randomized trial. J Thorac Cardiovasc Surg. 2019;158:895-907.

5. Sadeghi AH, Maat APWM, Taverne YJHJ, Cornelissen R, Dingemans A-MC, Bogers AJJC, et al. Virtual reality and artificial intelligence for 3-dimensional planning of lung segmentectomies. J Thorac Cardiovasc Surg Tech. 2021;7; 309-21. 